8-1-2021

\title{
Probabilistic measures of HIV-1 transmission in different HIV-1 key population groups of Larkana, Pakistan
}

Syed Rizwan-Ul-Hasa

Abiha Abdullah

Shakil Arshad Siddiqui

Shakil Ahmed

Sharaf Ali Shah

See next page for additional authors

Follow this and additional works at: https://ecommons.aku.edu/pakistan_fhs_mc_mc

Digipalrt of the Life Sciences Commons, Maternal and Child Health Commons, and the Pediatrics cemmens

Network 


\section{Authors}

Syed Rizwan-Ul-Hasa, Abiha Abdullah, Shakil Arshad Siddiqui, Shakil Ahmed, Sharaf Ali Shah, Fatima Mir, and Syed Hani Abidi 


\title{
Probabilistic measures of HIV-1 transmission in different HIV-1 key population groups of Larkana, Pakistan
}

\author{
Syed Rizwan-ul-Hasan, ${ }^{1}$ Abiha Abdullah, ${ }^{2}$ Shakil Ahmed, ${ }^{3}$ Sharaf Ali Shah,, Fatima Mir, ${ }^{5}$ Syed Hani Abidi6
}

\begin{abstract}
Objective: To estimate the probability of human immunodeficiency virus (HIV)-1 transmission from different key HIV population groups using probabilistic modelling.

Methods: This study was conducted in December 2020. A probabilistic model was used to estimate the probability of HIV-1 transmission from different key HIV population groups in Larkana. Our model was run on three probabilistic assumptions: 1) each replication gave two conceivable results: 'true' or 'false'; 2) the chance of giving a 'true' result is the same for each replication; and 3) the replications are independent - 'true' in one will not impact the likelihood of 'true' in another.

Results: The results estimated the probability of HIV transmission in key HIV population groups in Larkana to range between 0.42-0.54 per trial, where the highest probability of transmission was predicted for men who have sex with men (MSM; 0.54 per trial), followed by transgender (TG; 0.46 per trial) and people who inject drugs (PWID; 0.457 per trial).

Conclusion: Our results suggest that there is a high likelihood of HIV transmission by key population groups in Larkana, such as MSM, TG, and PWID. Mathematic models, such as one proposed in our study can aid the HIV and acquired immunodeficiency syndrome (AIDS) control programmes in evaluating and optimising the strategies in controlling transmission of HIV from the key population groups.

Keywords: HIV, Human immunodeficiency virus, Pakistan, Sexual and gender minorities, Transgender, Probabilistic, Larkana. (JPMA 71: S-26 [Suppl. 4]; 2021)
\end{abstract}

DOI: https://doi.org/10.47391/JPMA.0005

\section{Introduction}

Human Immunodeficiency Virus 1 (HIV-1) remains a major public health challenge worldwide. ${ }^{1}$ Globally, the number of new HIV cases has significantly decreased, due to an increase in usage of antiretroviral therapy (ART), and public health efforts to mitigate HIV transmission, such as needle-exchange programmes and $100 \%$ condom use per sex (CUPS), has resulted in a significant decrease in new HIV cases worldwide. For example, between 2000 and 2018, new HIV infections and HIV-related deaths decreased by $37 \%$ and $45 \%$, respectively. 2

In countries such as Pakistan, the number of new HIV-1 cases have proportionally increased over the past decade, with a nearly $57 \%$ increase in the number of new infections between 2010 and 2017.2 There have been eight documented HIV outbreaks in the past two decades. Four out of eight previous outbreaks occurred

1Department of Computer Sciences, DHA Suffa University, Karachi, 2Medical College, Aga Khan University, Karachi, ${ }^{3}$ Sir Syed University of Engineering and Technology, Karachi, 4Bridge Consultants Foundation, Karachi, 5 Department of Pediatrics and Child Health, ${ }^{6}$ Department of Biological and Biomedical Sciences, Aga Khan University, Karachi, Pakistan.

Correspondence: Syed Hani Abidi. Email: m.haniabidi@gmail.com in the Larkana district, suggesting that Larkana is an epicentre for HIV transmission in Sindh. In Larkana, the key HIV population groups include: people who inject drugs (PWID) accounting for $38 \%$ of currently registered HIV-positive cases; male sex workers (MSM) and female sex workers (FSW), the incidence of HIV among whom have projected to rise from $18.9 \%$ in 2015 to $24.3 \%$ in 2020 (among MSM) and from 3.2\% in 2015 to $6.3 \%$ in 2020 (among FSW); and finally transgender (TG) or hijra sex workers (HSW) in whom the HIV prevalence is around $27.6 \% .^{3-5}$ More recently Larkana witnessed another HIV outbreak in April 2019; more than 900 individuals, mostly children, have been identified as HIVpositive, making this outbreak the largest HIV outbreak in children in the world. ${ }^{3}$ In Larkana, and in Pakistan in general, health awareness and knowledge, particularly in rural areas, is low, and subsequently certain high-risk behaviours are prevalent, making high-risk population vulnerable to HIV transmission. As a result, there is a strong possibility that the overall prevalence of HIV is underreported in Pakistan. ${ }^{4}$ Additionally, isolated reports have documented bridging of the epidemic between high- and low-risk populations; however, it is not known which high-risk groups in Larkana are responsible or have a greater chance of spreading HIV-1 in the district, which can give rise to more HIV outbreaks 
in the future. ${ }^{5}$

Mathematical probabilistic models have been used previously to forecast HIV / acquired immunodeficiency syndrome (AIDS) transmissions. ${ }^{6}$ They assume the initial HIV burden to model the spread of HIV infection and predict future cases. ${ }^{7}$ One past study from Tanzania for example, applied a nonlinear mathematical model to study the effect of screening and treatment on transmission of HIV/AIDS infection in a population. ${ }^{8}$ In areas with high HIV transmission, mathematical probabilistic modelling can be useful in determining which key population groups have the greatest risk of HIV transmission; such information can aid in establishing effective interventional strategies.

In this study, we applied probabilistic modelling on available HIV-1 prevalence data on key population groups in Larkana to estimate which key HIV population groups in Larkana have the highest probability of transmitting HIV-1 infections.

\section{Methods}

\section{Prevalence data and assumptions}

This study was conducted in December 2020. Probabilistic modelling was applied to estimate the probability of HIV transmission by key population groups in Larkana, Pakistan. The modelling was performed assuming the probability of HIV transmission in TG, MSM, FSW, and people who inject drugs (PWID) to be 0.182 , $0.049,0.041$, and 0.162 , respectively, based on the reported prevalence of $18.2 \%, 4.9 \%, 4.1 \%$, and $16.2 \%$, among TG, MSM, FSW and PWID in Larkana, respectively. ${ }^{9-}$ 11 For the simulations, 302, 280, 349, and 364 trials were performed for PWID, TG, MSM, and FSW, respectively.

\section{Assumptions for the probabilistic model}

Our model was run on three probabilistic assumptions:

1. Each replication of the method comes about in one of two conceivable results: true or false;

2. The likelihood of 'true' is the same for each replication;

3. The replications are independent, meaning that a 'true' in one HIV-positive individual does not impact the likelihood of 'true' in another.

Probability $\mathrm{P}$ for $\mathrm{n}$ or more HIV positive individuals is the outcome.

\section{Probabilistic model:}

$\mathrm{P}\left(\right.$ "n or more HIV positive individuals") $=1-\sum_{n=0}^{x}(m n) P^{n}(1-P)^{m-n}$ Where "n", "m" and "P" are as follows:
- "n" number of HIV positive individuals;

- "m" is the population or trials; and

- "P" is the probability of $\mathrm{n}$ or more HIV positive individuals.

\section{Results}

We applied probabilistic modelling to estimate the probability of HIV transmission by key population groups in the Larkana, Pakistan. The modelling was based on the reported prevalence of HIV in the key population group, where the highest prevalence was found to be in TG followed by PWIDs.

Overall, the probability of HIV transmission in key HIV population groups of Larkana was found to be between 0.42-0.54 per trial $(302,280,349$, and 364 trials for PWID, TG, MSM, and FSW, respectively; Figure-1). Contrary to the reported prevalence, the highest probability of transmission was predicted for MSM (0.54 per trial [349]), followed by TG (0.46 per trial [280]) and PWID (0.457 per trial [302]) (Figure-1). Since our model worked on the

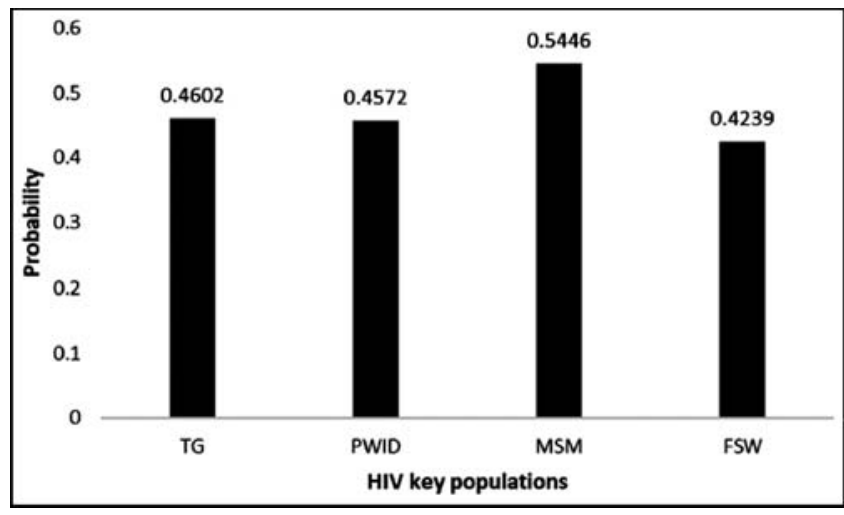

Figure-1: Binomial probability for human immunodeficiency virus (HIV) transmission in Larkana: Graph shows the probability of HIV transmission in different HIV key populations in Larkana, Pakistan, namely people who inject drugs (PWID), transgender (TG), men who have sex with men (MSM), and female sex workers (FSW). As indicated earlier, simulations were performed using 302, 280, 349, and 364 trials, respectively.

assumption that replications are independent of each other, meaning that the likelihood of acquiring HIV by one individual does not impact the likelihood of acquisition in another, the probabilistic measures of our study predict MSMs to be the major driver of HIV transmission in Larkana.

\section{Discussion}

This study aimed to use probabilistic modelling to estimate the chances of HIV-1 transmission by different key HIV population groups of Larkana, Pakistan. 
Probabilistic modelling was conducted through $M{ }^{2} T_{L A B}{ }^{\circledR}$ software. Our results suggest that key population groups in Larkana have a high HIV transmission probability, implying that if HIV is not controlled in these key population groups, Larkana can soon become a major driver of HIV transmission in Pakistan.

As indicated from the four key HIV population groups in Larkana, the highest probability of HIV-1 transmission (0.54) was estimated for MSM, followed by TG and PWIDs. These findings are in agreement with the published reports that identified that in Pakistan prevalence of HIV-1 in the general population is very low $(0.04 \%)$, but very high in high-risk groups such as PWIDs (36.4\%), HSWs (12\%), MSMs (17.5\%), and sex workers (53.5\%). ${ }^{12}$ The increased prevalence of HIV in key population groups in Larkana is attributed to several factors, such as unemployment, poverty, lack of parental supervision, peer pressure and poor infection control practices, etc. ${ }^{13}$ Another important factor for the steady rise of HIV among PWID is lack of proper needle exchange programmes in the district. ${ }^{13}$ Moreover, Larkana has functioning brothels, where FSWs receive clients not only from the district but nearby cities as well. Additionally, there are also at least two motels on Larkana's Station Road which is located in the middle of the city where hijra (transgender) and MSW are available round the clock, which might be a probable cause of the high HIV prevalence amongst MSM, HSWs and FSWs in this region. ${ }^{14}$ In contrast to female sex workers, women in the general population are considered to be at low risk for HIV since they do not exhibit or indulge in highrisk practices. ${ }^{15}$ However in Pakistan, highly stigmatised MSMs often marry women to conceal their homosexual identity from society; their sexual relationships with their wives lead to HIV transmission to their wives. ${ }^{13,15}$ Additionally, children born out of this relationship can be HIV-positive. ${ }^{15}$

We anticipate few limitations of our analysis. First, to keep the outcomes as realistic as possible, the modelling was based on the reported prevalence of HIV in key population groups of Larkana. Underreported prevalence may likely lead to incorrect projections and the actual transmission probability can be higher than projected. Second, all probabilities were calculated assuming an equal chance of transmission, while it is possible that in reality some groups would have higher chances of transmitting HIV than others. These limitations can be addressed with dynamic mathematical modelling in follow-ups studies as more data from Larkana become available, providing a more robust estimate of HIV in different high-risk groups.

In conclusion, our current study, corroborating the finds of previous studies, suggest that the key HIV population groups in Larkana are likely to transmit HIV within the key population groups and possibly beyond the key populations to low-risk communities. In such a scenario the likelihood of getting new HIV cases is high over the next few years. 3 Mathematic models, such as one proposed in our study, can aid the National/Provincial AIDS control programmes in evaluating and optimising the strategies in controlling transmission of HIV from the key population groups.

Disclaimer: None.

\section{Conflicts of Interest: None.}

Funding: This study was funded by the Higher Education Commission Grant No. 5217/Sindh/NRPU/R\&D/HEC/2016 and Pakistan Science Foundation Grant No. PSF/Res/SAKU/Med (488).

\section{References}

1. Tariq U, Iftikhar A, Zahid D, Sultan F, Mahmood SF, Naeem S, et al The emergence of an unassigned complex recombinant form in a Pakistani HIV-infected individual. Arch Virol 2020;165:967-72. doi: 10.1007/s00705-020-04551-4.

2. UNAIDS. Global HIV \& AIDS Statistics - 2019 Fact Sheet [Internet] 2019 [cited 2020 Dec 16]. Available from URL: https://www.unaids.org/en/resources/fact-sheet.

3. Mir F, Mahmood F, Siddiqui AR, Baqi S, Abidi SH, Kazi AM, et al. HIV infection predominantly affecting children in Sindh, Pakistan, 2019: a cross-sectional study of an outbreak. Lancet Infect Dis 2020;20:362-70. doi: 10.1016/S14733099(19)30743-1.

4. The Lancet Infectious Diseases. HIV epidemics in Pakistan. Lancet Infect Dis 2019;19:671. doi: 10.1016/S1473-3099(19)30316-0.

5. Khanani MR, Somani M, Rehmani SS, Veras NM, Salemi M, Ali SH. The spread of HIV in Pakistan: bridging of the epidemic between populations. PLoS One 2011; 6:e22449. doi: 10.1371/journal.pone.0022449.

6. Lee S, Ko J, Tan X, Patel I, Balkrishnan R, Chang J. Markov chain modelling analysis of HIV/AIDS progression: a race-based forecast in the United States. Indian J Pharm Sci 2014;76:107-15.

7. Cassels S, Clark SJ, Morris M. Mathematical models for HIV transmission dynamics: tools for social and behavioral science research. J Acquir Immune Defic Syndr 2008;47(Suppl 1):S34-9. doi: 10.1097/QAl.0b013e3181605da3.

8. Safiel R, S Massawe ES, Makinde DO. Modelling the effect of screening and treatment on transmission of HIV/AIDS infection in a population. American Journal of Mathematics and Statistics 2012;2:75-88. doi: 10.5923/j.ajms.20120204.03

9. Waheed $\mathrm{Y}$, Waheed H. Pakistan needs to speed up its human immunodeficiency virus control strategy to achieve targets in fast-track acquired immune deficiency syndrome response. World J Virol 2017;6:46-8. doi: 10.5501/wjv.v6.i2.46.

10. Emmanuel F, Adrien A, Athar U, Imran M, Reza T, Blanchard J. Using surveillance data for action: lessons learnt from the second generation HIV/AIDS surveillance project in Pakistan. East 
Mediterr Health J 2011;17:712-8.

11. Hasan Z, Shah S, Hasan R, Rao S, Ahmed M, Stone M, et al. Late diagnosis of human immunodeficiency virus infections in highrisk groups in Karachi, Pakistan. Int J STD AIDS 2018;29:1400-6. doi: 10.1177/0956462418785264.

12. Singh S, Ambrosio M, Semini I, Tawil O, Saleem M, Imran M, et al. Revitalizing the HIV response in Pakistan: a systematic review and policy implications. Int J Drug Policy 2014;25:26-33. doi: 10.1016/j.drugpo.2013.05.011.
13. Altaf A, lqbal S, Shah SA. A third major human immunodeficiency viruses (HIV) outbreak in Larkana, Pakistan: caused by unsafe injection practices. J Pak Med Assoc 2019;69:1068-9.

14. Memon A, Haider S, Altaf A. Alarming increase in reported HIV cases from Larkana, Pakistan: a matter of serious concern. J Pak Med Assoc 2014;64:205-6.

15. Raees MA, Abidi SH, Ali W, Khanani MR, Ali S. HIV among women and children in Pakistan. Trends Microbiol 2013;21:213-4. doi: 10.1016/j.tim.2012.12.005. 